\title{
Creation of 2-5 keV and 5-10 keV sky maps using XMM-Newton data
}

\author{
D. O. Savchenko ${ }^{1}$, D. A. Iakubovskyi ${ }^{1,2}$ \\ ${ }^{1}$ Bogolyubov Institute of Theoretical Physics, Metrologichna str. 14-b, 03680, Kyiv, Ukraine \\ ${ }^{2}$ National University "Kyiv-Mohyla Academy", Skovorody str. 2, 04070, Kyiv, Ukraine \\ dsavchenko@bitp.kiev.ua
}

\begin{abstract}
Sky maps are powerful visualisation tools for quicklook analysis of extended sources. The latest sky map in soft $\mathrm{X}$-rays (0.1-2.4 keV) has been created in 90ies using ROSAT data. By analyzing publically available data from $X M M$ Newton X-ray mission we constructed new sky maps in two energy bands - 2-5 keV and 5-10 keV, complementary to ROSAT data, covering about $1 \%$ of all sky, and included them to our web-based tool http://skyview.virgoua.org
\end{abstract}

Key words: X-rays: general, virtual observatory tools

\section{INTRODUCTION}

Usually, astronomers deal with catalogues of point sources. However, if the source is extended (i.e. its size is comparable or even bigger than the point spread function of the instrument), more sophisticated method of scientific data visualisation is needed. The most common method of such visualisation is building sky maps - specially processed series of 2-dimensional images in different energy bands. An example of such map for X-ray astronomy is all-sky map in 0.1-2.4 keV band made by ROSAT X-ray satellite [1, 2] observations. This all-sky map also exists as interactive web-tool [3].

After the end of ROSAT mission, several missions in $\mathrm{keV}$ range have been operating. These missions have covered a minor part of the sky (not more than several \%) but with much better sensitivity and wider energy range compared with ROSAT. In this paper, we present the interactive maps in 2-5 and 5-10 keV range. For these maps, we use publicly available observations by MOS cameras [4] of XMM-Newton [5] X-ray mission. Special attention was paid to handle with most important background components, including soft proton flares and quiescent particle background, see [6] for detailed properties of XMM-Newton background. The obtained map is included to website of Virtual Roentgen and Gamma Observatory in Ukraine $\square$.

\section{METHODS}

For constructing sky map, we first downloaded all publically available (on July 1, 2013) observation data files for MOS [4] cameras of XMM-Newton X-ray observatory [5] available on the HEASARC data archive [8]. These data files were processed using Extended Sources Analysis Software (ESAS) package [9, 10] specially developed for analysis of extended sources at the NASA/GSFC XMM-Newton Guest Observer Facility 11 in cooperation with the XMM-Newton Science Operation Centre [12] and the XMM-Newton Background Working Group [13]. It is publically available as part of XMM-Newton Science Analysis System (SAS) v.13.5.0. The methodology of ESAS software is based on detailed modeling and/or subtraction of various background components (see [6] for complete list) experienced by MOS and PN cameras onboard XMM-Newton cosmic mission using the "first principles" as much as possible. To model instrumental

${ }^{1}$ http://skyview.virgoua.org

Table 1: General properties of MOS observations used in our analysis.

\begin{tabular}{ccc}
\hline Camera & MOS1 & MOS2 \\
\hline No. of observations & 3942 & 4022 \\
No. of data files & 4029 & 4104 \\
Cleaned exposure, Ms & 77.9 & 81.5 \\
\hline
\end{tabular}




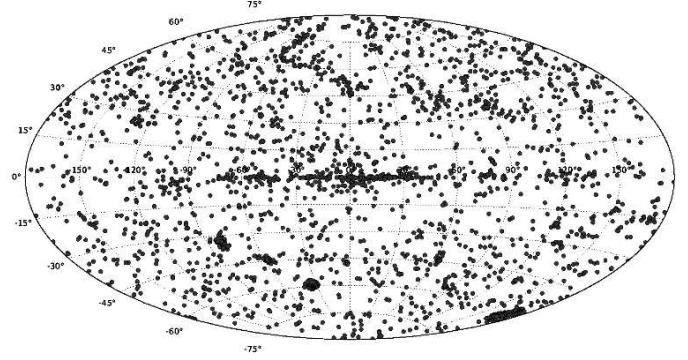

Fig. 1: Positions (in galactic coordinates) of $X M M-$ Newton observations used in our analysis. The fieldof-views of XMM-Newton observations are given in natural values, so one can easily recognize the zones observed by XMM-Newton covering about $1 \%$ of all sky.

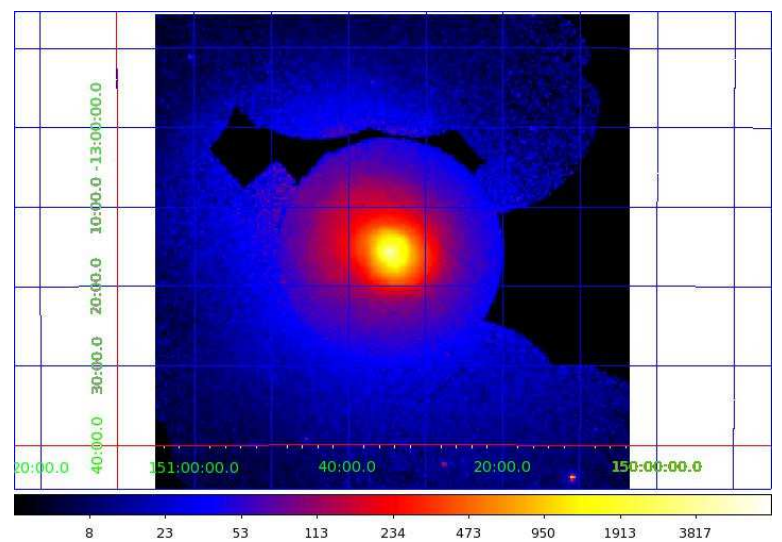

Fig. 3: A 2-5 keV image of 1 square degree around Perseus cluster of galaxies. The units are in $\operatorname{cts} / \mathrm{s} / \operatorname{deg}^{2}$.

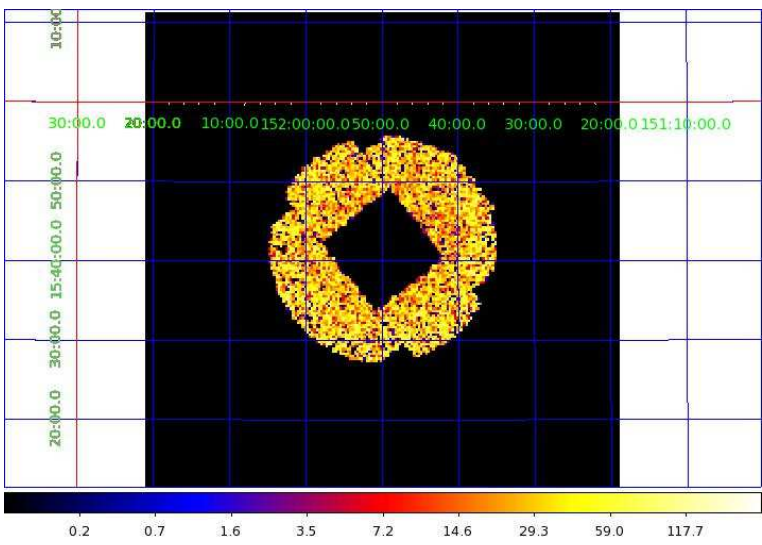

Fig. 2: An example of very bright point source observation - polar BY Cam in 2-5 keV. The units are in $\mathrm{cts} / \mathrm{s} / \mathrm{deg}^{2}$. The position of BY Cam coincides with central CCD of MOS instruments. Because this point source is very bright ( $\gtrsim 1 \mathrm{mCrab}[7]$ ), it was not observed in usual imaging mode and therefore appears as a "gap" in the sky map.

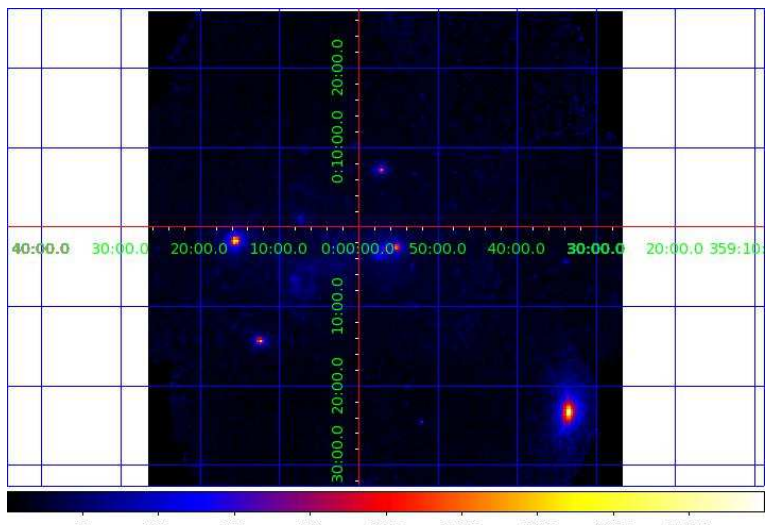

Fig. 4: The same as at the left Figure but for Milky Way centre in 5-10 keV range.

background, ESAS software relies on filter-wheel-closed data and the data from the unexposed corners of archived observations, rather than "blank sky" data (contaminated by unknown level by different variable background components) used by a number of other methods. This is essential for analysis of very faint sky regions (e.g. galaxy cluster outskirts) dominated by the background (rather than the source) emission. The obtained data products - filtered event ${ }^{2}$ lists, images, lightcurves and spectra - are produced in FITS [15, 16] format for user-defined regions within XMM-Newton field-of-view.

Our data reduction is started from production of filtered event lists using ESAS script mos-filter. This script effectively removes time intervals affected by highly variable background component - soft proton flares, see 6, 10. We used the standard filters and cuts provided by ESAS software. For example, we selected single, double, triple and quadruple events (described by event PATTERN $<=12$ ) of highest quality

\footnotetext{
${ }^{2}$ Here, an "event" is a result of instantaneous positive detection in one or several adjacent CCD pixels. Single photon hitting the CCD may produce substantial signal in adjacent pixels causing so-called multuple (e.g. double, triple, quadruple) events. The standard selection procedure used in our analysis takes into account single, double, triple and quadruple events for MOS cameras. According to 14 the procedure based on analysis of event patterns allows to reject of about $99 \%$ of events caused by high-energy $(\sim 100 \mathrm{MeV})$ cosmic rays thus significantly reducing the amount of data telemetry.
} 
(described by FLAG $==0$ ) 3 . Main parameters for obtained event lists are shown in Table 1, The leftover MOS event lists were processed by ESAS scripts mos-spectra and mos_back giving observed and modelled quiscent particle background spectra, exposure maps, count images for selected energy ranges and modelled particle background count images. The resulting images and exposure maps of individual observations are then combined by ESAS scripts merge_comp_xmm and bin_image_merge into count-rate images of sky regions with size $22^{\circ} \times 22^{\circ}$ and minimal pixel size $2.5^{\prime \prime} \times 2.5^{\prime \prime}$. Point sources are not excluded, although very bright point sources observed with timing mode (such as BY Cam, see Fig. 2) haven't been processed by ESAS and therefore do not appear in our map. For the sky map, we chose two energy ranges $-2-5 \mathrm{keV}$ and $5-10 \mathrm{keV}-$ motivated by their

- negligible contamination by remaining Solar Wind Charge Exchange background component, see [6, 10] for details;

- complementarity to existing ROSAT all-sky map [3] in 0.1-2.4 keV.

For sky map visualisation, we used the standard NASA skyview.jar tool [19]. This tool selects appropriate images overlapping with given sky region and samples them to the given pixel size. The SutherlandHodgman clipping algorithm was used to resample images. This method treats the output pixel grid as a window over the input images grid and integrates the flux within each output pixel exactly. The output image can be produced in given sky coordinates and projection. The obtained images in FITS [15, 16] format are available for quick look and can be directly downloaded from http://skyview.virgoua.org.

\section{RESULTS}

We constructed sky maps in $2-5 \mathrm{keV}$ and $5-10 \mathrm{keV}$ bands using $\sim 4000$ publically available observations of MOS cameras on-board XMM-Newton X-ray cosmic mission. Positions of given observations and their basic properties are shown in Fig. 1 and Table 1 respectively. The produced maps are cleaned from variable soft proton component and instrumental background with the help of standard analysis for extended sources ESAS software 9] - and included to web-interface of Virtual Roentgen and Gamma-Ray Observatory in Ukraine, http://skyview.virgoua.org, see Figs 3 and 4 as examples. The obtained maps cover about $1 \%$ of all sky, see Fig. 1 for details. They are complementary to existing ROSAT all-sky map in soft X-rays (0.1-2.4 keV) as well as usual X-ray catalogues of point sources.

\section{ACKNOWLEDGEMENT}

We thank Yuri Izotov, Vladimir Savchenko, Igor Telezhinsky, Ievgen Vovk and the anonymous Referee for their comments and suggestions. This work was supported by part by the Program of Cosmic Research of the National Academy of Sciences of Ukraine, the State Programme of Implementation of Grid Technology in Ukraine and the grant of President of Ukraine for young scientists.

\section{REFERENCES}

[1] S. L. Snowden, M. J. Freyberg, P. P. Plucinsky, J. H. M. M. Schmitt, J. Truemper, W. Voges, R. J. Edgar, D. McCammon and W. T. Sanders, First Maps of the Soft X-Ray Diffuse Background from the ROSAT XRT/PSPC All-Sky Survey, ApJ 454 (Dec., 1995) 643.

[2] W. Voges, B. Aschenbach, T. Boller, H. Bräuninger, U. Briel, W. Burkert, K. Dennerl, J. Englhauser, R. Gruber, F. Haberl, G. Hartner, G. Hasinger, M. Kürster, E. Pfeffermann, W. Pietsch, P. Predehl, C. Rosso, J. H. M. M. Schmitt, J. Trümper and H. U. Zimmermann, The ROSAT all-sky survey bright source catalogue, A\&A 349 (Sept., 1999) 389-405 astro-ph/9909315.

[3] "Rosat x-ray background tool." http://heasarc.gsfc.nasa.gov/cgi-bin/Tools/xraybg/xraybg.pl.

44 M. J. L. Turner, A. Abbey, M. Arnaud, M. Balasini, M. Barbera, E. Belsole, P. J. Bennie, J. P. Bernard, G. F. Bignami, M. Boer, U. Briel, I. Butler, C. Cara, C. Chabaud, R. Cole, A. Collura, M. Conte, A. Cros, M. Denby, P. Dhez, G. Di Coco, J. Dowson, P. Ferrando, S. Ghizzardi, F. Gianotti, C. V. Goodall, L. Gretton, R. G. Griffiths, O. Hainaut, J. F. Hochedez, A. D. Holland, E. Jourdain,

\footnotetext{
${ }^{3}$ Such selection based on FLAG keyword excludes events out of instrument FoV, near CCD corners and "hot pixels" etc. It is generally recommended [17, 18] to select FLAG $==0$ for high-quality spectral analysis.
} 
E. Kendziorra, A. Lagostina, R. Laine, N. La Palombara, M. Lortholary, D. Lumb, P. Marty, S. Molendi, C. Pigot, E. Poindron, K. A. Pounds, J. N. Reeves, C. Reppin, R. Rothenflug, P. Salvetat, J. L. Sauvageot, D. Schmitt, S. Sembay, A. D. T. Short, J. Spragg, J. Stephen, L. Strüder, A. Tiengo, M. Trifoglio, J. Trümper, S. Vercellone, L. Vigroux, G. Villa, M. J. Ward, S. Whitehead and E. Zonca, The European Photon Imaging Camera on XMM-Newton: The MOS cameras : The MOS cameras, A\&A 365 (Jan., 2001) L27-L35 arXiv:astro-ph/0011498.

[5] F. Jansen, D. Lumb, B. Altieri, J. Clavel, M. Ehle, C. Erd, C. Gabriel, M. Guainazzi, P. Gondoin, R. Much, R. Munoz, M. Santos, N. Schartel, D. Texier and G. Vacanti, XMM-Newton observatory. I. The spacecraft and operations, A\&A 365 (Jan., 2001) L1-L6.

[6] "Xmm-newton epic background components." http://www.star.le.ac.uk/ amr30/BG/BGTable.html.

7] W. H. Baumgartner, J. Tueller, C. B. Markwardt, G. K. Skinner, S. Barthelmy, R. F. Mushotzky, P. A. Evans and N. Gehrels, The 70 Month Swift-BAT All-sky Hard X-Ray Survey, ApJS 207 (Aug., 2013) 19 [1212.3336.

[8] "Heasarc: Nasa's archive of data on energetic phenomena." http://heasarc.nasa.gov.

9] "Cookbook for analysis procedures for xmm-newton epic observations of extended objects and the diffuse background." http://heasarc.gsfc.nasa.gov/docs/xmm/esas/cookbook/xmm-esas.html.

[10] K. D. Kuntz and S. L. Snowden, The EPIC-MOS particle-induced background spectra, A\&A 478 (Feb., 2008) 575-596.

[11] "Nasa/gsfc xmm-newton guest observer facility." http://heasarc.gsfc.nasa.gov/docs/xmm/xmmgof.html.

[12 "Xmm-newton science operation centre." http://xmm.esac.esa.int

13 "Xmm-newton background working group." http://xmm2.esac.esa.int/external/xmm_sw_cal/background

[14] D. H. Lumb, R. S. Warwick, M. Page and A. De Luca, X-ray background measurements with XMM-Newton EPIC, A\&A 389 (July, 2002) 93-105 [arXiv:astro-ph/0204147.

[15] E. W. Greisen and M. R. Calabretta, Representations of world coordinates in FITS, A\&A 395 (Dec., 2002) 1061-1075 astro-ph/0207407.

[16] M. R. Calabretta and E. W. Greisen, Representations of celestial coordinates in FITS, A\&A 395 (Dec., 2002) 1077-1122 astro-ph/0207413.

[17] E. Piconcelli et. al., XMM-Newton Users Handbook, issue 2.10. ESA: XMM-Newton SOC, 2012. http://xmm.esac.esa.int/external/xmm_user_support/documentation/uhb.

[18] 1. de la Calle et. al., Users Guide to the XMM-Newton Science Analysis System, issue 10.5. ESA: XMM-Newton SOC, 2014. http://xmm.esac.esa.int/external/xmm_user_support/documentation/sas_usg/USG.

[19] "Skyview in a jar." http://skyview.gsfc.nasa.gov/current/jar/skyviewinajar.html. 